\title{
announcements
}

\section{Nominations Sought for 2002 Sullivan and Perlman Awards}

The American Geophysical Union (AGU) is seeking nominations for two annual journalism awards: the Walter Sullivan Award for Excellence in Science Journalism-Features and the David Perlman Award for Excellence in Science Journalism-News. These awards consist of a plaque and a $\$ 2,000$ stipend and will be presented at AGU's 2002 Spring or Fall Meeting.

These awards recognize work that enhances public awareness and understanding of the sciences encompassed by AGU: the study of the earth, the sun, the solar system, and their environments and components. The Perlman Award is for work produced under deadline pressure of one week or less, while the Sullivan Award is for work produced with longer lead time. Work in any medium, except books, is eligible, as long as it was intended for and available to the general public. Work from any country and in any language is eligible. (Entries not originally in English must include an English translation.) AGU has adopted several rule changes for the 2002 awards, resulting from suggestions made both by journalists and the independent judging committee, which recommends winners to the AGU Council. These are as follows:

- Eligibility dates and deadline: The entire process has been advanced by approximately two weeks, in order to allow the judging committee sufficient time to consider the growing number of entries. For the 2002 awards, entries must first have been published between 16 December 2000 and 15 December 2001. Entries must be received at AGU no later than 31 December 2001.

- One entry per person for each award: Previously, a journalist could be nominated for up to three articles (broadcasts, etc.) for each award. Since the award is for a single publication, not a body or work, this often resulted in votes being split among several excellent submissions by the same author and thus working to the detriment of that author. For the 2002 awards, therefore, a limit of one entry per person for each award has been instituted (i.e., one may still be nominated for both the Sullivan and Perlman Awards). If a journalist is nominated by third parties for more than one work, AGU will contact the nominee to determine which one entry should be retained.

- Multiple authors: The previous rules prevented many collaborative efforts from being judged, as only one person could be deemed the author of an entry. That restriction has been removed: now those involved in a collaborative effort will decide who is or are the nominee(s). Only one plaque and one $\$ 2,000$ stipend will be provided for each award.

- Series redefined: As in the past, up to three items may be submitted as a single entry, if they constituted, or were part of, a series. A "series" was defined as a group of segments on a common theme and identified as such at the time of publication. This remains true for the Sullivan Award, but for the Perlman Award, the definition has been expanded. In addition to the above, up to three items may be submitted that constituted coverage of an ongoing event-for example, a scientific society meeting, an earthquake, a meteorological phenomenon.

- Third party nominations encouraged: While anyone could make nominations for these awards in past years, in practice, almost all entries were self-nominations. The judging committee believes that this was because the submission requirements (e.g., six copies of an article or videotape, an English translation) could be onerous for those other than the nominated journalists themselves. Therefore, for other than self-nominations, nominators will be asked only for one copy of a work they believe worthy of consideration for an award. AGU will then contact the authors and advise them of the nominations. If they wish the nomination to go forward, they will be required to submit the necessary materials prior to the 31 December deadline.

This announcement is a summary of some of the rules for these awards, with emphasis on changes. The only authoritative statement of all relevant rules is on the AGU Web site at http://www.agu.org/sci_soc/ 
sci_awards.html. A nomination form and list of previous winners of these awards may also be accessed from that page.

\section{Gill Instruments Announces Addition of New Anemometer}

Gill Instruments Ltd., electronics and ultrasonic anemometer specialists, announces a new addition to their extension, the R3-50.

Designed in response to customer requests, the R350 employs the latest electronic components combined with Gill's experience in producing ultrasonic anemometers for the micrometeorological research market. Complementing the existing range of three-axis research anemometers, the R3-50 uses advances from the mass-produced WindMaster Pro and software from the R3, to offer the $50 \mathrm{~Hz}$ sensor.

With no moving parts, the R3-50 is virtually maintenance free, requires no calibration, and provides an ideal solution for users doing research with slow large eddies at high mast levels. Having identical output formats and sharing the same operating software as the R3, the R3-50 is particularly suited to applications where a complete wind profile is required; with an R3 positioned near to the ground and the R3-50 positioned to a higher level. The R3-50 is rigorously tested to internationally recognized standards and meets the stringent performance criteria specified by meteorological, naval, and airport authorities and oil and utility companies around the world.

\section{Fellowships in Atmospheric Science and Re- lated Research}

The Cooperative Institute for Research in Atmosphere at Colorado State University (CIRA) offers a limited number of one-year associate fellowships to research scientists, including those on sabbatical leave or recent Ph.D. recipients. Those receiving the awards will pursue their own research programs, collaborate with existing programs, and participate in institute seminars and functions. Selection is based on the likelihood of an active exchange of ideas between the fellows, the National Oceanic and Atmospheric Administration (NOAA), Colorado State University, and CIRA scientists. Salary is negotiable based on experience, qualification, and funding support. The program is open to scientists of all countries. Submitted applications should include a curriculum vitae, publications list, brief outline of the intended research, a statement of estimated research support needs, and names and addresses of three professional references.

CIRA is jointly sponsored by Colorado State University and NOAA. Colorado State University is an equal opportunity employer and complies with all Federal and Colorado state laws, regulations, and executive orders regarding affirmative action requirements. In order to assist Colorado State University in meeting its affirmative action responsibilities, ethnic minorities, women, and other protected class members are encouraged to apply and to so identify themselves. Senior scientists and qualified scientists from foreign countries are encouraged to apply and to combine the CIRA stipend with support they receive from other sources.

Applications for positions that begin 1 January are accepted until the prior 31 October and should be sent to Thomas G. Vonder Haar, Director CIRA, Colorado State University, Fort Collins, CO 80523. Research fellowships are available in the areas of air quality, cloud physics, mesoscale studies and forecasting, satellite applications, climate studies, model evaluation, and economic and societal aspects of weather and climate. For more information, visit the Web site at www.cira.colostate.edu. 